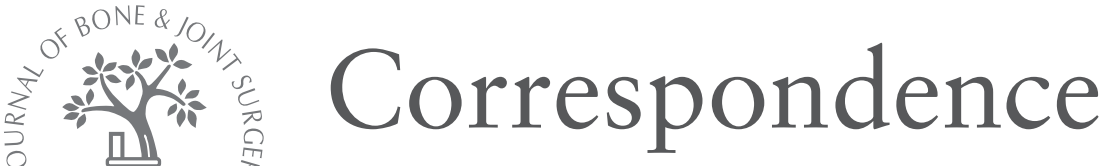

We welcome letters to the Editor concerning articles which have recently been published. Such letters will be subject to the usual stages of selection and editing; where appropriate the authors of the original article will be offered the opportunity to reply.

Letters should normally be under $\mathbf{3 0 0}$ words in length, doublespaced throughout, signed by all authors and fully referenced. The edited version will be returned for approval before publication.

(C)2006 British Editorial Society of Bone and Joint Surgery \$2.00 J Bone Joint Surg [Br] 2006;88-B:137-8.

\section{Factors associated with nonunion in conservatively- treated type-II fractures of the odontoid process}

Sir

We read with interest the article by Koivikko et $\mathrm{al}^{1}$ in the November 2004 issue entitled 'Factors associated with nonunion in conservatively-treated type-II fractures of the odontoid process'. The purpose of the study was to identify the risk factors which increase the probability of not achieving bony union in patients with typeII fractures of the odontoid process who are treated conservatively in a halo vest. Although the paper is based only on patients who were treated conservatively, we agree with the majority of the arguments regarding the different factors involved in the possible development of a type-II fracture of the odontoid process to a pseudarthrosis.

There are, however, many factors involved in the prognosis of a fracture of the odontoid process that have to be considered. All these factors have been studied either separately ${ }^{2}$ or in conjunction. ${ }^{3,4}$ Each one is important, so all have to be considered. In a study from our institution we classified all these factors and introduced the concept of a fracture of the odontoid process as being at 'risk of pseudarthrosis'. 3

Furthermore, recent work on the peripheral quantitative CT scan analysis of the axis vertebra to show its internal architecture and structural properties ${ }^{5}$ revealed that this is the most important factor to be taken into account when dealing with these fractures. It is strongly related to the age of the patients and also to type-II fractures.

doi:10.1302/0301-620X.88B1.17343

D. S. KORRES, MD

G. D. CHLORES, MD

G. S. THEMISTOCLEOUS, MD

Kat Hospital,

Athens, Greece.

1. Koivikko MP, Kiuru MJ, Koskinen SK, et al. Factors associated with nonunion in conservatively-treated type-II fractures of the odontoid process. J Bone Joint Surg [Br] 2004:86-B:1146-51.

2. Apuzzo ML, Heiden JS, Weiss MH, et al. Acute fractures of the odontoid process: an analysis of 45 cases. J Neurosurg 1978;48:85-91.

3. Korres DS, Stamos K, Andreakos A, Hardouvelis C, Kouris A. Fractures of the dens and risk of pseudarthrosis. Arch Orthop Trauma Surg 1989;108:373-6.
4. Roy-Camille R, Saillant G, Lapresle P, Leonard P. Recent fractures of the odontoid: prognostic factors. Presse Med 1983;12:2233-6.

5. Korres DS, Karachalios T, Roidis N, et al. Structural properties of the axis studied in cadaveric specimens. Clin Orthop 2004:418:134-40.

\section{Author's reply:}

Sir,

I would like to thank Drs Korres, Chloros and Themistocleous for their interest in our paper. We agree that the aetiology of nonunion appears to be multifactorial. In multivariate analysis we found that patient age was statistically unrelated to nonunion when simultaneously controlling for the effects of the remaining risk factors. Similar to ordinary univariate analysis, confident demonstration of a zero effect using multivariate analysis requires a large sample size. Therefore, based on our relatively limited material it would only be valid to state that we found age to be a non-significant predictor of nonunion. To the best of our knowledge, none of the studies which conclude that higher incidences of nonunion in elderly patients have statistically compensated for the confounding effects by multiple risk factors.

Studies on quantitative and qualitative micro-architecture of the odontoid process ${ }^{1}$ provide an important scientific basis both for understanding these injuries and for future use of the rapidly developing diagnostic imaging methods. Multidetector CT scans can easily provide detailed information on odontoid fracture morphology ${ }^{2}$ and we are in no doubt that in the future even more detailed information will be obtained and used clinically. However, to date, no clinical studies using micro-architecture to predict nonunion exist. More research needs to be done before microarchitectural variations can be associated with nonunion. doi:10.1302/0301-620X.88B1.17344

M. P. KOIVIKKO, MD, PhD

Helsinki University Hospital, Helsinki, Finland.

1. Amling M, Hahn M, Wening VJ, Grote HJ, Delling G. The microarchitecture of the axis as the predisposing factor for fracture of the base of the odontoid process: a histomorphometric analysis of twenty-two autopsy specimens. J Bone Joint Surg [Am] 1994;76-A:1840-6.

2. Koivikko MP, Kiuru MJ, Koskinen SK. Occurrence of comminution (type IIA) in type II odontoid process fractures: a multi-slice CT study. Emerg Radiol 2003;10:84-6.

\section{Methicillin-resistant Staphylococcus aureus in trauma and orthopaedic practice}

Sir,

We read with interest the review article by Giannoudis, Parker and Wilcox ${ }^{1}$ in the June 2005 issue entitled 'Methicillin-resistant Staphylococcus aureus in trauma and orthopaedic practice'.

The scourge of Methicillin-resistant Staph. aureus (MRSA) is one of the biggest challenges facing modern orthopaedics in the United Kingdom. It is of the utmost importance that the medical profession as a whole works together to combat the spread of MRSA, and the 
suggested protocol for prevention and treatment should be strictly adhered to by all hospitals. It is correctly stated that "the focus must be on adequate prophylaxis" and that "hand hygiene is thought to be the cornerstone of the prevention of MRSA infection". In view of this we feel that the main priority is the need to enforce effective hand hygiene within the hospital environment. Compliance with regulations has traditionally been poor, but this cannot be tolerated. In a study of our staff we found that the technique of hand disinfection with alcohol-based gel was generally ineffective, although following training with an ultraviolet light box and fluorescent gel performance improved significantly. ${ }^{2}$ Regular training and testing of staff should be part of normal clinical practice and hand washing should be strictly enforced by an ethos of open observation.

While hand washing dispensers at every bed space may overcome some of these issues there needs to be a concerted effort to change practices. It should be unacceptable for staff not to wash their hands before and after contact with every patient. Discussion of the prevention of MRSA and hand hygiene will help to draw attention to the importance of this issue.

doi:10.1302/0301-620X.88B1.17026

D. J. MACDONALD, MBChB, BSc, MRCS

A. J. R. GRAY, FRCSEd(Orth)

Western Infirmary,

Glasgow, UK.
1. Giannoudis PV, Parker J, Wilcox MH. Methicillin-resistant Staphylococcus aureus in trauma and orthopaedic practice. J Bone Joint Surg [Br] 2005;87-B: 749-54.

2. Macdonald DJM, Mckillop ECA, Trotter S, Gray AJR. Hand washing in the orthopaedic department: a cross over study of hand washing performance. Ann R Coll Surg Eng/ 2005; (in press).

\section{Author's reply:}

Sir,

We would like to thank Messrs Macdonald and Gray for their interest in our paper. Prevention of Methicillin-resistant Staphylococcus aureus should be of the utmost importance. It is clear that compliance with regulations for hand hygiene has been poor over the years. As stated in our manuscript some of the reasons for noncompliance include lack of knowledge or forgetfulness, understaffing and heavy workloads. The policy of placing hand-hygiene dispensers at every bed and around the wards has been helpful. However, the strategy for the prevention should also include frequent educational meetings and audit cycles. This should overcome the issue of poor compliance.

doi:10.1302/0301-620X.88B1.17027

P. V. GIANNOUDIS, BSc, MD, EEC(Orth)

St James's University Hospital,

Leeds, UK. 